Dr. Deborah Cook MD FRCPC MSC (Epid)

\section{Systematic reviews: the case for rigorous methods and rigorous reporting}

preventive, therapeutic, rehabilitative and palliative interventions. Thus, narrative reviews may be most useful for obtaining a broad overview of a topic or for describing cutting edge developments in a field; they are less often useful in furnishing precise answers to specific clinical questions.

A systematic review, in contrast to a traditional, narrative non-systematic review article, includes a clear statement of the purpose of the review, a comprehensive search and retrieval of the relevant research, explicit selection criteria, critical appraisal of the primary studies, and reproducible decisions regarding relevance, selection, and methodological rigour of the primary research. ${ }^{6}$ If results of primary studies are summarized quantitatively in a systematic review, the article is called a meta-analysis.

Does it really matter whether clinicians read non-systematic or systematic reviews? The answer is yes! Traditional reviews are much more likely to yield incomplete or biased information. Moreover, without systematic reviews, the dissemination of effective treatment may be delayed up to a decade, and the endorsement of harmful therapy may persist for years after its harm has been clearly demonstrated; this was the case with the use of thrombolytic therapy and lidocaine, respectively, for acute myocardial infarction. ${ }^{7}$ Moreover, the epidemiology of review articles is changing rapidly; the number of systematic reviews published annually has increased at least 500-fold in the past decade. ${ }^{8,9}$ It is not unusual to find more than one systematic review addressing the same or a very similar therapeutic question. ${ }^{10}$ Readers need to become familiar with the merits and demerits of different types of review articles, and how to interpret them best.

Accordingly, the publication in this issue of the Canadian Journal of Anaesthesia by Smith highlights some important points about the review articles found in four leading ànaesthesia journals. When evaluated using standard critical appraisal guides, the style of the 
reviews and/or their reporting structure were found wanting in several domains. Consumers of the literature have trouble assessing the validity of reviews, and also their usefulness in practice, if the review methods are not rigorous or if they are not clearly reported. As Smith comments, the responsibility for creating high quality systematic reviews and qualitative syntheses (metaanalyses), when appropriate, rest not only with reviewers themselves but also with the peer review system.

Readers may benefit from both narrative and systematic reviews, and labelling them as such is important. Since the original audits of the biomedical literature, other disciplines have been examining review articles in their own literature ${ }^{11}$ and coming up with similar conclusions to those by Smith. Many journals are now considering only publishing systematic review articles, and standardizing a transparent reporting style of the review methods. For example, modern day systematic review articles ideally contain a methods section, with subtitles including "search strategy, selection criteria, methodological quality assessment, data abstraction, data analysis (if a quantitative synthesis occurs and this section is appropriate)."

In the future, widespread use of computers will improve the accessibility of reviews for clinicians, administrators and policy-makers. More efficient search methods to identify systematic reviews will be developed. Clinicians will become more skilled in critically appraising reviews. ${ }^{12}$ Presentation formats will become more user-friendly for both providers and their patients. New practical techniques for incorporating information from systematic reviews into clinical decision making will be created and disseminated. The study by Smith in this issue should prompt action in this direction.

Meanwhile, readers interested in reviews may become more familiar with the international initiative called the Cochrane Collaboration, which is helping to prepare, maintain and disseminate the results of systematic reviews of health care interventions. ${ }^{13}$ The Cochrane Data Base of Systematic Reviews is the first large scale, multidisciplinary product of the collaboration, containing 65 reviews, 53 protocols, 168 titles of additional reviews in preparation, a bibliography of methodological articles about the science of reviewing research, and information on the Cochrane Collaboration. ${ }^{14}$ The second issue is now available on disc on CD-ROM, in Windows, Macintosh and DOS formats, and is being distributed by the American College of Physicians, the Canadian Medical Association and the British Medical Journal. Topics reviewed to date under the auspices of the Cochrane Collaboration span many disciplines, including anaesthesia.

\section{Les articles de revues : vers des méthodes plus rigoureuses et des comptes rendus plus précis}

Habituellement, les cliniciens se renseignent en lisant des d'articles originaux ou des travaux de synthèse dans les journaux médicaux. Les premiers font le compte rendu d'une recherche originale ; les travaux de synthèse sont des condensés d'investigations publiés sous la forme de revues générales, de directives d'exercice, d'analyses économiques et décisionnelles.

Pour le clinicien, la lecture des articles originaux de recherche peut représenter une tâche insurmontable. En 1980, on a estimé que la masse des écrits biomédicaux s'accroissait de 6 à $7 \%$ par année et doublait ainsi tous les 10 à 12 ans. ${ }^{1}$ En se basant sur un calcul effectué en 1979, on sait que 220000 articles biomédicaux paraissent chaque année ; de là, on a déterminé que si un clinicien lisait deux articles par jour pendant toute une année, il accumulerait à la fin de l'année un retard de 55 siècles ! ' De nos jours, environ 25000 journaux biomédicaux contenant des centaines d'articles chacun sont imprimés annuellement. ${ }^{3}$

Parmi les avantages procurés par la lecture de comptes rendus de travaux originaux, il faut mentionner le fait qu'il sont récents, qu'ils sont soumis à une certaine forme de sélection (le jugement des pairs) et qu'ils sont accessibles sur ordinateur. Par contre leur usage est peu commode, ils occupent beaucoup d'espace et coûtent cher. ${ }^{4}$ Comme toutes les sources de renseignements, les articles principaux et complémentaires doivent être abordés avec un esprit critique avant que leurs conclusions ne s'appliquent au domaine des soins de santé. Lorsqu'il s'agit de trouver une solution à un problème clinique spécifique, l'article original de bonne qualité demeure souvent le seul recours valable. Toutefois, s'il doit choisir entre plusieurs articles originaux (quelquefois en désaccord) sur un sujet important, le clinicien devrait favoriser les articles de revue systématiques.

Les articles traditionnels de revue s'adressent à plusieurs aspects spécifiques d'un sujet donné. ${ }^{3} \mathrm{Par}$ exemple, une revue sur le diabète (par ex., le type chapitre de manuel) comprendra des sections sur la physiologie et la physiopathologie du métabolisme des 
hydrates de carbone, des lipides et des protéines, l'épidémiologie et le pronostic, les approches diagnostiques et les moyens de prévention, le traitement et les interventions palliatives. Ce type de revue permet d'obtenir une vue d'ensemble sur un sujet particulier ou encore de mettre à jour ses connaissances sur un développement marquant ; il est toutefois moins adapté à fournir une solution précise à un problème clinique spécifique.

L'article de revue systématique, contrairement à l'article traditionnel, fournit un énoncé clair de son objectif, inclut la méthode de la recherche et du recueil complets des travaux pertinents, des critères explicites de sélection, une évaluation critique des études initiales et des conclusions reproductibles sur la pertinence, la sélection et la rigueur méthodologique de la recherche originale. ${ }^{6}$ Dans une revue systématique, si les résultats des études originales sont rapportés en tenant compte de leur qualité, l'article devient alors une méta-analyse.

Est-il plus avantageux pour le clinicien de lire des articles systématiques de revue ? La réponse est oui. Les articles de revue traditionnels sont plus sujets à livrer de l'information incomplète et biaisée. De plus, hors du cadre systématique, la transmission d'une thérapeutique efficace peut accuser une décennie de retard, alors qu'une pratique médiocre peut se perpétuer pendant des années même une fois sa nocivité démontrée ; c'est ce qui est survenu pour le traitement de l'infarctus du myocarde avec la thrombolyse et la lidocaïne. ${ }^{7}$ En outre, l'épidémiologie des articles de revue est en perpétuel changement ; le nombre des revues systématiques publiées annuellement a augmenté d'au moins 500 fois au cours de la dernière décennie. ${ }^{8,9}$ Il n'est pas rare de trouver plus d'une revue systématique publiée sur une question thérapeutique ou sur un problème connexe..$^{10}$ Les lecteurs doivent se familliariser avec les mérites et les défauts des différents types d'articles de revue et avec la meilleure façon de les interpréter.

À ce sujet, la publication de Smith dans ce numéro du Journal canadien d'anesthésie nous éclaire sur certains aspects très importants des articles de revue publiés dans les quatre principaux journaux d'anesthésie de langue anglaise. Lorsque évalués avec des critères critiques standards, leur rédaction et leur structure étaient déficients dans plusieurs domaines. Si la méthode de vérification manque de rigueur et le compte rendu de précision, les lecteurs ont autant de difficulté à en évaluer leur validité que leur pertinence sur le plan clinique. Comme Smith le fait remarquer, la responsabilité de produire des revues systématiques et des synthèses qualitatives (méta-analyse) de bonne qualité, selon le cas, n'incombait pas uniquement à ceux qui soumettaient l'article de revue, mais aussi au mécanisme de jugement par les pairs.

Les lecteurs peuvent bénéficier aussi bien des revues narratives que des revues systématiques, mais leur identification comme telles devient importante. Depuis le démarrage de l'audit de la littérature biomédicale, plusieurs autres disciplines ont adopté la même politique pour leurs propres publications ${ }^{11}$ et en sont arrivées aux mêmes conclusions que Smith. Plusieurs journaux considèrent maintenant ne publier que des articles de revues systématiques et tentent de standardiser une formule transparente pour leur réalisation. Par exemple, les article systématiques modernes de revue contiennent de préférence une section portant sur les méthodes avec des sous-titres tels que stratégie de recherche, critères de sélection, évaluation de la qualité de la méthodologie, la façon d'extraire les données et leur analyse (pour une synthèse qualitative et sa pertinence).

À l'avenir, la diffusion par l'informatique assurera l'accessibilité des revues aux cliniciens, aux administrateurs et aux décideurs. Des méthodes plus efficaces permettant d'identifier les articles systématiques de revue seront développées. Les cliniciens deviendront plus aptes à les évaluer avec un esprit critique. ${ }^{12} \mathrm{La}$ présentation deviendra plus conviviale autant que pour celui qui donne les soins que pour celui qui les reçoit. Des méthodes pratiques permettant de faire le pont entre l'information fournie par les revues systématiques et les décisions cliniques seront créées. L'étude de Smith publiée ici marque un progrès dans ce sens.

Entre-temps, les lecteurs que les revues intéressent peuvent se familiariser avec l'initiative surnommée Collaboration Cochrane, très utile pour préparer, conserver et diffuser les résultats de revues systématiques en soins de santé. ${ }^{13} \mathrm{La}$ base des données de revues systématiques de Cochrane constitue les premiers résultats d'une collaboration multidisciplinaire réalisée sur une vaste échelle, contenant 65 revues, 53 protocoles, 168 titres additionnels de revues en préparation, une bibliographie de la méthodologie de recherche nécessaire à la réussite d'un article de revue et des renseignements sur la Collaboration Cochrane. ${ }^{14}$ La deuxième édition est disponible sur disque optique compatible avec les formats Windows, Macintosh et DOS et est distribuée par l'American College of Physicians, l'Association médicale canadienne et le British Medical Journal. Les sujets passés en revue par la Collaboration Cochrane couvrent plusieurs disciplines dont l'anesthésie.

\section{References}

1 Sackett DL. How to read clinical journals: why to read them and how to start reading them critically. Can Med Assoc J 1981; 124: 555-8. 
2 Bernier CL, Yekery AN. Cogent communication: overcoming information overload. Westport, Connecticut: Greenwood Press 1979: 39.

3 Marshall JG. The impact of the hospital library on clinical decision making: the Rochester Study. Bull Med Libr Assoc 1992; 80: 169-78.

4 Haynes RB, McKibbon KA, Fitzgerald D, Guyatt GH, Walker CJ, Sackett $D L$. How to keep up with the medical literature. I. Why try to keep up and how to get started. Ann Intern Med 1986; 105: 149-53.

5 Mulrow $C D$. The medical review article: state of the science. Ann Intern Med 1987; 106: 485-8.

6 Cook DJ, Sackett DL, Spitzer WO. Methodological guidelines for systematic reviews of randomized control trials in health care from the Potsdam Consultation on Meta-analysis. J Clin Epidemiol 1995; 48: 167-71.

7 Antman EM, Lau J, Kupelnick B, Mosteller F, Chalmers TC. A comparison of results of meta-analyses of randomized control trials and recommendations of clinical experts. Treatments for myocardial infarction. JAMA 1992; 268: 240-8.

8 Chalmers TC, Lau J. Meta-analytic stimulus for changes in clinical trials. Stat Meth Med Res 1993; 2 : 161-72.

9 Chalmers I, Haynes RB. Reporting, updating, and correcting systematic reviews of the effects of health care. In: Chalmers I, Altman DG (Eds.). Systematic Reviews. London: BMJ Publishing Group; 1995 : 86-95.

10 Jadad A, Cook DJ, Browman G. When arbitrators disagree: resolving discordant meta-analysis. Can Med Assoc J 1997; (in press).

11 Auperin A, Pignon JP, Poynard T. Critical review of meta-analyses of randomized clinical trials in gastroenterology. Aliment Pharmacol Ther 1997; (in press).

12 Oxman $A D$, Cook DJ, Guyatt GH, and the EvidenceBased Medicine Working Group. Users' guides to the medical literature. VI. How to use an overview. JAMA 1994; 272 : 1367-71.

13 Chalmers $I$. The Cochrane Collaboration: preparing, maintaining and disseminating systematic reviews of the effects of health care. In: Warren KS, Mosteller F (Eds.). Doing More Good than Harm: The Evaluation of Health Care Interventions. New York: Annals of the New York Academy of Sciences, 1993: 156-63.

14 Cochrane News. Issue Number 3. ISSN 1324-1257. Oxford, UK. May 1995. 